\title{
An Inequality for Fermion-Systems
}

\section{H. Nicolai*}

Institut für theoretische Physik, Universität Karlsruhe, D-7500 Karlsruhe, Federal Republic of Germany

\begin{abstract}
It is shown that for certain classes of Euclidean fermion-boson systems on a lattice vacuum expectation values of scalar fields increase if a Yukawa-interaction is turned on. Applicability and possible extensions of this result in the framework of constructive quantum-field-theory are discussed.
\end{abstract}

\section{Introduction}

In recent times much of the progress made in constructive field theory has been due to the extensive use of correlation inequalities such as the Griffiths and Lebowitz inequalities ([1,2] where original work is quoted). However, until now, the range of applicability of this powerful tool appears to have been limited to purely scalar theories. In this paper I want to show that by applying Griffiths' inequality one can derive an inequality for systems containing not only a scalar or $^{1}$ a pseudoscalar field but also a Majorana- or ${ }^{1}$ Dirac-spinor.

To state the main result some notation must be introduced that will be used in the sequel. As the UV-limit will not be considered in this article the models are formulated on a periodic Euclidean space-time lattice $\mathscr{T}^{2}$ with lattice-spacing $a$ whose elements will be denoted by $k, m, n, \ldots$ where $n=\left(n_{0}, n_{1}, n_{2}, n_{3}\right)$; because of periodic boundary conditions there exists $N \in \mathbb{N}$ such that $n_{\mu}$ and $n_{\mu}+N$ are to be identified. Then the type of theory I will consider contains the following parts:

1) Bosonic part of the action

$$
\begin{aligned}
& S_{0}(A)=a^{4} \sum_{n \in \mathscr{T}}\left\{\frac{Z_{0}}{2}\left(\nabla_{\mu} A_{n}\right)^{2}+\frac{m_{0}^{2}}{2} A_{n}^{2}+\lambda A_{n}^{4}\right\} \\
& S_{0}(B)=a^{4} \sum_{n \in \mathscr{T}}\left\{\frac{Z_{0}}{2}\left(\nabla_{\mu} B_{n}\right)^{2}+\frac{m_{0}^{2}}{2} B_{n}^{2}+\lambda B_{n}^{4}\right\}^{3},
\end{aligned}
$$

* Supported by Studienstiftung des deutschen Volkes

1 Exclusive "or"

2 For simplicity, I restrict myself to the case $d=4$. Most of the results of this paper can be recovered for $d=2$ and $d=3$ in a straightforward manner

$3 \nabla_{\mu} \varphi_{n}:=\frac{1}{2 a}\left(\varphi_{n+\hat{\mu}}-\varphi_{n-\hat{\mu}}\right)$ 
where $A$ is scalar and $B$ is pseudoscalar; $Z_{0}>0$ (wave-function-renormalization parameter), $m_{0}^{2} \in \mathbb{R}$ and $\lambda>0$. Vacuum-expectation-values with respect to either of the above theories will be denoted by $\langle\ldots\rangle_{0}$. Observe that this measure obeys Griffith's inequalities: it is an even ferromagnet [2].

2) Fermionic part of the action ${ }^{4}$

$$
\begin{aligned}
& S_{1}\left(A, \psi^{(2)}, \psi^{(1)}\right)=a^{4} \sum_{n \in \mathscr{T}}\left\{Z_{1} \psi_{n}^{(2)} \gamma^{\mu} \nabla^{\mu} \psi_{n}^{(1)}+m_{1} \psi_{n}^{(2)} \psi_{n}^{(1)}+g A_{n} \psi_{n}^{(2)} \psi_{n}^{(1)}\right\} \\
& S_{1}\left(B, \psi^{(2)}, \psi^{(1)}\right)=a^{4} \sum_{n \in \mathscr{T}}\left\{Z_{1} \psi_{n}^{(2)} \gamma^{\mu} \nabla^{\mu} \psi_{n}^{(1)}+g B_{n} \psi_{n}^{(2)} \gamma^{5} \psi_{n}^{(1)}\right\}
\end{aligned}
$$

where $\psi_{n}^{(2)}, \psi_{n}^{(1)}$ are Osterwalder-Schrader Euclidean Fermi-fields [3]; $Z_{1}>0, m_{1}$, $g \in \mathbb{R}$. One can also take $\psi_{n}^{(2)}$ and $\psi_{n}^{(1)}$ to be Euclidean Majorana-spinors, i.e. $\psi_{n}^{(2)}=\mathscr{C} \psi_{n}^{(1)}$ where $\mathscr{C}$ is the charge-conjugation matrix [4]; in this case $Z_{1}, m_{1}, g$ have to be replaced by $\frac{1}{2} Z_{1}, \frac{1}{2} m_{1}, \frac{1}{2} g$. Vacuum-expectation-values with respect to $S_{0}+S_{1}$ will be denoted by $\langle\ldots\rangle_{1}$.

Then the main result may be stated as follows.

Theorem. Let $G, H$ be any functions of $A$ (or $B$ ) fulfilling the assumptions for Griffiths inequalities to hold [2]. Then, with the above assumptions

$$
\langle G H\rangle_{1} \geqq\langle G\rangle_{1}\langle H\rangle_{0} \geqq\langle G\rangle_{0}\langle H\rangle_{0} \text {. }
$$

Taking $H \equiv 1$, it follows in particular that

$$
\langle G\rangle_{1} \geqq\langle G\rangle_{0} \geqq 0 \text {. }
$$

In Section 2, a preliminary lemma giving sufficient conditions for (1.3) and (1.4) to be satisfied will be proven. In Section 3, the proof of the theorem will be given by verifying the assumptions of this lemma. Finally, in Section 4, possible applications and extensions of the main theorem will be discussed.

\section{Some Preliminary Remarks}

Since only vacuum-expectation-values of (pseudo) scalar fields will be considered the fermions can be "integrated out"-explicitly by treating them as elements of a Grassmann-algebra and using Berezin's integral on Grassmann-algebras [5]. The result is

Lemma 2.1. (Matthews-Salam-Formula [6]).

(i) If $\psi_{n}^{(1)}, \psi_{n}^{(2)}$ is a Dirac-spinor

$$
\begin{aligned}
& \int e^{-S_{1}\left(A, \psi^{(2)}, \psi^{(1)}\right)} \prod_{n \in \mathscr{T}} \prod_{\alpha=1}^{4} d \psi_{n, \alpha}^{(1)} d \psi_{n, \alpha}^{(2)} \\
& \quad=a^{16|\mathscr{T}|} \operatorname{det}\left\{Z_{1} \gamma_{\alpha \beta}^{\mu} \nabla_{k n}^{\mu}+\delta_{\alpha \beta} \delta_{k n}\left(m_{1}+g A_{n}\right)\right\}^{5}
\end{aligned}
$$

and analogously for a pseudoscalar.

4 I choose the following representation of $\gamma$-matrices:

$$
\begin{aligned}
\gamma^{\mu} & =\left(\begin{array}{cc}
0 & \hat{\sigma}^{\mu} \\
\hat{\sigma}^{\mu+} & 0
\end{array}\right)=\gamma^{\mu+}, \quad \hat{\sigma}^{\mu}:=\left(1, i \sigma^{k}\right), \quad \gamma^{5}=\left(\begin{array}{cc}
i & 0 \\
0 & -i
\end{array}\right)=-\left(\gamma^{5}\right)^{+} \\
5 & \left.\nabla_{k n}^{\mu}=\frac{1}{2 a}\left(\delta_{k+\hat{\mu}, n}-\delta_{k-\hat{\mu}, n}\right) \text { (matrix element of } \nabla^{\mu}\right)
\end{aligned}
$$


ii) If $\psi_{n}^{(2)}=\mathscr{C} \psi_{n}^{(1)}$ is a Euclidean Majorana-spinor and if the replacement $Z_{1} \rightarrow \frac{1}{2} Z_{1}$, etc. is made in (1.2)

$$
\begin{aligned}
& \int e^{-S_{1}\left(A, \psi^{(2)}, \psi^{(1)}\right.} \prod_{n \in \mathscr{T}} \prod_{\alpha=1}^{4} d \psi_{n, \alpha}^{(1)} \\
& \quad=a^{8|\mathscr{T}|}\left[\operatorname{det}\left\{Z_{1} \gamma_{\alpha \beta}^{\mu} \nabla_{k n}^{\mu}+\delta_{\alpha \beta} \delta_{k n}\left(m_{1}+g A_{n}\right)\right\}\right]^{1 / 2}
\end{aligned}
$$

and analogously for a pseudoscalar.

Remark. The proof of (i) is rather straightforward. The proof of (ii) is a little more complicated; the validity of (2.2) depends on the antisymmetry of the matrices $\left(\mathscr{C} \gamma^{\mu}\right)_{\alpha \beta} \nabla_{k n}^{\mu}, \mathscr{C}_{\alpha \beta} \delta_{k n}$ and $\left(\mathscr{C} \gamma^{5}\right)_{\alpha \beta} \delta_{k n}$ (pseudoscalar case) in an essential way. Up to a numerical factor this formula can be found e.g. in [7]. Writing $Z_{1} x_{n}:=m_{1}+g A_{n}$ and $Z_{1} y_{n}:=g B_{n}$, I now consider the following functions

$$
\begin{array}{ll}
F_{1}\left(\left\{x_{n}\right\}\right):=\operatorname{det}\left\{\gamma_{\alpha \beta}^{\mu} \nabla_{k n}^{\mu}+\delta_{\alpha \beta} \delta_{k n} x_{n}\right\}, & x_{n} \in \mathbb{R}, \\
F_{2}\left(\left\{y_{n}\right\}\right):=\operatorname{det}\left\{\gamma_{\alpha \beta}^{\mu} \nabla_{k n}^{\mu}+\gamma_{\alpha \beta}^{5} \delta_{k n} y_{n}\right\}, & y_{n} \in \mathbb{R} .
\end{array}
$$

When dealing with Majorana-spinors the relevant quantities are $F_{1}^{1 / 2}$ and $F_{2}^{1 / 2}$. Since the lattice $\mathscr{T}$ is finite it is clear that the functions $F_{1}$ and $F_{2}$ are polynomials in $x_{n}, n \in \mathscr{T}$ where each $x_{n}$ occurs at most four times in each monomial. Thus

$$
F_{1}\left(\left\{x_{n}\right\}\right)=\sum_{\varrho=1}^{4|\mathscr{T}|} \sum_{\substack { \Gamma_{1}, \ldots, \Gamma_{4} \subset \mathcal{T} \\
\Gamma_{2} \cap \Gamma_{j}=\phi \text { if } i \neq j \\
\begin{subarray}{c}{\sum_{i=1}\left|\Gamma_{2}\right|=\varrho{ \Gamma _ { 1 } , \ldots , \Gamma _ { 4 } \subset \mathcal { T } \\
\Gamma _ { 2 } \cap \Gamma _ { j } = \phi \text { if } i \neq j \\
\begin{subarray} { c } { \sum _ { i = 1 } | \Gamma _ { 2 } | = \varrho } }\end{subarray}}\left[c_{\left\{\Gamma_{i}\right\}} \prod_{i=1}^{4} \prod_{n \in \Gamma_{\imath}} x_{n}^{i}\right]
$$

and similarly for $F_{2}\left(\left\{y_{n}\right\}\right)$ [in (2.5) the term with $\varrho=0$ vanishes because $\left.\operatorname{det}\left(\gamma_{\alpha \beta}^{\mu} \nabla_{k n}^{\mu}\right)=0\right]$.

From (2.3) and (2.4) it may not be seen that $F_{1}^{1 / 2}$ and $F_{2}^{1 / 2}$ are also polynomials but this follows from the representation formula (2.2), so in that case an expression similar to $(2.5)$ can be written down. The following identity is an immediate consequence of (2.1).

$$
\langle G\rangle_{1}=\frac{\langle G F\rangle_{0}}{\langle F\rangle_{0}}
$$

As will be shown below, $\langle F\rangle_{0}>0$, so (2.6) is well defined. Note that I have introduced the shorthand notation

$$
F=\sum_{\left\{\Gamma_{i}\right\}} c_{\left\{\Gamma_{i}\right\}} M_{\left\{\Gamma_{i}\right\}},
$$

where $F=F_{1}$ or $F_{2}$ is understood and $M_{\left\{\Gamma_{t}\right\}}\left(\left\{x_{n}\right\}\right)$ is the monomial $\prod_{i=1}^{4} \prod_{n \in \Gamma_{i}} \mathrm{x}_{n}^{i}$.

Lemma 2.2. If $c_{\left\{\Gamma_{i}\right\}} \geqq 0^{6}$ for all $\left\{\Gamma_{i}\right\}$ and if $G$ and $H$ are functions of $A$ (or $B$ ) fulfilling the assumptions for Griffiths' inequalities

$$
\langle G H\rangle_{1} \geqq\langle G\rangle_{1}\langle H\rangle_{0} \geqq\langle G\rangle_{0}\langle H\rangle_{0} .
$$

6 In the scalar case $Z_{1} x_{n}=m_{1}+g A_{n}$ and one has to require $m_{1} g>0$ (consider $m_{1}, g>0$ and $<0$ separately); the case $m_{1} g<0$ is equivalent to $m_{1} g>0$ by $A \rightarrow-A$ symmetry 
Proof. Because of Griffiths' inequality

$$
\begin{aligned}
\langle G H F\rangle_{0} & =\sum_{\left\{\Gamma_{i}\right\}} c_{\left\{\Gamma_{i}\right\}}\left\langle G H M_{\left\{\Gamma_{i}\right\}}\right\rangle_{0} \\
& \geqq \sum_{\left\{\Gamma_{i}\right\}} c_{\left\{\Gamma_{i}\right\}}\left\langle G M_{\left\{\Gamma_{i}\right\}}\right\rangle_{0}\langle H\rangle_{0}=\langle G F\rangle_{0}\langle H\rangle_{0}
\end{aligned}
$$

( $M_{\left\{\Gamma_{i}\right\}}$ obeys Griffiths' inequality being a monomial).

Thus

$$
\langle G H\rangle_{1}=\frac{\langle G H F\rangle_{0}}{\langle F\rangle_{0}} \geqq \frac{\langle G F\rangle_{0}}{\langle F\rangle_{0}}\langle H\rangle_{0}=\langle G\rangle_{1}\langle H\rangle_{0}
$$

The second inequality in (2.8) is obtained by repeating this estimate. Also, by Griffiths' inequality, $\langle F\rangle_{0} \geqq 0$ and, observing that $c_{\{\phi, \phi, \phi, \mathscr{T}\}}>0$, in fact $\langle F\rangle_{0}>0$. It is remarkable that $F\left(\left\{x_{n}\right\}\right) \geqq 0$ is not necessary to derive the latter inequality.

The bulk of the proof therefore consists in proving $c_{\left\{\Gamma_{\nu}\right\}} \geqq 0$ for all $\left\{\Gamma_{i}\right\}$. This will be done in the next section.

\section{Proof of Positivity of Coefficients}

To make this proof more transparent only the case (2.3) will be considered in detail. To do so, I introduce a function $P\left(\left\{x_{n, \alpha}\right\}\right)$ of $4|\mathscr{T}|$ variables $x_{n, \alpha} \in \mathbb{R}(n \in \mathscr{T}$, $\alpha=1, \ldots, 4)$

$$
P\left(\left\{x_{n, \alpha}\right\}\right):=\operatorname{det}\left\{\gamma_{\alpha \beta}^{\mu} \nabla_{k n}^{\mu}+\delta_{\alpha \beta} \delta_{k n} x_{k, \alpha}\right\} .
$$

If the assumptions made in Lemma 2.2 can be proven to hold for $P\left(\left\{x_{n, \alpha}\right\}\right)$ they are true a fortiori for $F\left(\left\{x_{n}\right\}\right)=P\left(\left\{x_{n} \delta_{\alpha \beta}\right\}\right)$.

Proposition 3.1. For $\left\{x_{n, \alpha}\right\} \in \mathbb{R}^{4|\mathscr{T}|} P\left(\left\{x_{n, \alpha}\right\}\right)$ is real.

Proof.

$$
\begin{aligned}
P^{*}\left(\left\{x_{n, \alpha}\right\}\right) & =\operatorname{det}\left\{-\gamma_{\alpha \beta}^{\mu} \nabla_{k n}^{\mu}+\delta_{\alpha \beta} \delta_{k n} x_{k, \alpha}\right\} \\
& =\operatorname{det}\left\{\left(i \gamma^{5}\right)_{\alpha \varrho}\left(-\gamma_{\varrho \tau}^{\mu} \nabla_{k n}^{\mu}+\delta_{\varrho \tau} \delta_{k n} x_{k, \tau}\right)\left(i \gamma^{5}\right)_{\tau \beta}\right\} \\
& =\operatorname{det}\left\{\gamma_{\alpha \beta}^{\mu} \nabla_{k n}^{\mu}+\delta_{\alpha \beta} \delta_{k n} x_{k, \alpha}\right\}=P\left(\left\{x_{n, \alpha}\right\}\right) .
\end{aligned}
$$

In the first line use was made of the fact that $\gamma^{\mu}$ is hermitean and $\nabla_{k n}^{\mu}$ is antihermitean; to go from the second to the third line it is essential that $i \gamma_{\alpha \beta}^{5}$ and $x_{n, \alpha} \delta_{\alpha \beta}$ commute (being diagonal matrices).

To formulate the next proposition the open set $G$ is introduced.

$$
G:=\left\{\left\{x_{n, \alpha}\right\} \in \mathbb{R}^{4|\mathscr{T}|} \text { and } x_{n, \alpha}>0 \text { for all } n \in \mathscr{T}, \alpha=1, \ldots, 4\right\} \text {. }
$$

Proposition 3.2. $P\left(\left\{x_{n, \alpha}\right\}\right)>0$ for all $\left\{x_{n, \alpha}\right\} \in G$.

Proof. Assume that there exists $\left\{y_{n, \alpha}\right\} \in G$ such that $P\left(\left\{y_{n, \alpha}\right\}\right) \leqq 0$. For large $t>0$ the dominating term in $P\left(\left\{t y_{n, \alpha}\right\}\right)$ is $t^{4|\mathscr{T}|} \prod_{n, \alpha} y_{n, \alpha}>0$; thus there exists $t_{0} \geqq 1$ such that 
$\left\{z_{n, \alpha}\right\}=\left\{t_{0} y_{n, \alpha}\right\} \in G$ and $P\left(\left\{z_{n, \alpha}\right\}\right)=0$ which is equivalent to

$$
\operatorname{det}\left\{i \gamma_{\alpha \beta}^{\mu} \nabla_{k n}^{\mu}+i \delta_{\alpha \beta} \delta_{k n} z_{k, \alpha}\right\}=0
$$

the factor $i$ is inserted so as to make $i \gamma_{\alpha \beta}^{\mu} \nabla_{k n}^{\mu}$ a hermitean matrix). Multiplying by the positive matrix $\left(z_{k, \alpha}\right)^{-1 / 2} \delta_{k n} \delta_{\alpha \beta}$ from left and right, it follows from (3.4) that

$$
\operatorname{det}\left\{\frac{1}{\sqrt{z_{k, \alpha}}}\left(i \gamma_{\alpha \beta}^{\mu} \nabla_{k n}^{\mu}\right) \frac{1}{\sqrt{z_{n, \beta}}}+i \delta_{\alpha \beta} \delta_{k n}\right\}=0^{7}
$$

which is a contradiction as $-i$ cannot be eigenvalue of a hermitean matrix !

In complete analogy to (2.4) one can write

$$
P\left(\left\{x_{n, \alpha}\right\}\right)=\sum_{\varrho=1}^{4|\mathscr{T}|} \sum_{\substack{\Delta_{1}, \ldots, \Delta_{4} \mathcal{C} \mathcal{T} \\\left|\Delta_{1}\right|+\ldots+\left|\Delta_{4}\right|=\varrho}}\left[c_{\{\Delta \alpha\}} \prod_{\alpha=1}^{4} \prod_{n \in \Delta_{\alpha}} x_{n, \alpha}\right],
$$

where now, in contradistinction to (2.5), each $x_{n, \alpha}$ appears once at most in each monomial.

Proposition 3.3. For all $\Delta_{\alpha} \subset \mathscr{T}(\alpha=1, \ldots, 4)$

$$
c_{\left\{\Delta_{\alpha}\right\}} \geqq 0 .
$$

(Actually, one can even show that $c_{\left\{\Delta_{\alpha}\right\}}=0$ if $\sum_{\alpha}\left|\Delta_{\alpha}\right|$ is odd.)

Proof. Choose $\tilde{\Delta}_{\alpha} \subset \mathscr{T}$ arbitrarily. Define

$$
G \ni x_{n, \alpha}^{\tilde{J}}:=\left\{\begin{array}{lll}
t & \text { if } & n \in \tilde{\Delta}_{\alpha} \\
t^{-1} & \text { if } & n \in \mathscr{T} \backslash \tilde{\Delta}_{\alpha}
\end{array}(t>0) .\right.
$$

Then

$$
P\left(\left\{x_{n, \alpha}^{\tilde{\Delta}}\right\}\right)=c_{\left\{\tilde{\Delta}_{\alpha}\right\}} t^{|\tilde{\Delta}|}+O\left(t^{|\tilde{\Delta}|-1}\right)^{8} .
$$

To prove (3.9), one has to distinguish the following cases:

$$
\Delta \subset \tilde{\Delta}, \Delta \supset \tilde{\Delta}, \Delta \cap \tilde{\Delta}=\emptyset, \Delta \nsubseteq \tilde{\Delta} \text { and } \tilde{\Delta} \nsubseteq \Delta \text { but } \Delta \cap \tilde{\Delta} \neq \emptyset \text {. }
$$

It then follows easily that the only term that contributes to the coefficient of $t^{|\tilde{\Delta}|}$ is $c_{\{\tilde{\Delta}\}}$. Assuming $c_{\{\tilde{U}\}}<0$ one arrives at a contradiction upon choosing $t$ sufficiently large because for all $t<\infty P\left(\left\{x_{n, \alpha}^{\tilde{A}}\right\}\right)>0$. This completes the proof of the proposition and thus, by Lemma 2.2, of the main theorem for scalar fields and Diracspinors.

\section{Defining}

$$
\tilde{P}\left(\left\{y_{n, \alpha}\right\}\right):=\operatorname{det}\left\{\gamma_{\alpha \beta}^{\mu} \nabla_{k n}^{\mu}+\gamma_{\alpha \beta}^{5} \delta_{k n} y_{k, \alpha}\right\}, \quad y_{n, \alpha} \in \mathbb{R}
$$

the proof for pseudoscalar fields runs along the same lines : reality of (3.10) follows directly because $\left(\gamma^{5}\right)^{+}=-\gamma^{5}$; positivity for $\left\{y_{n, \alpha}\right\} \in G$ from

$$
\tilde{P}\left(\left\{y_{n, \alpha}\right\}\right)=\operatorname{det}\left\{-i\left(\gamma^{5} \gamma^{\mu}\right)_{\alpha \beta} \nabla_{k n}^{\mu}+i \delta_{\alpha \beta} \delta_{k n} y_{k, \alpha}\right\}
$$

\footnotetext{
7 No summation on indices $\alpha, \beta, k, n$ !

$8 \quad|\tilde{\Delta}|:=\Gamma^{4}|\Delta$.
} 
and the hermiticity of the matrix $i\left(\gamma^{5} \gamma^{\mu}\right)_{\alpha \beta} \nabla_{k n}^{\mu}$. The proof of Proposition 3.3 is the same as before.

To make the proof of the main theorem complete, the case of Majoranaspinors remains to be investigated. To do so, I make a special choice of $\left\{x_{n, \alpha}\right\}$ :

$$
\hat{x}_{n, \alpha} \delta_{\alpha \beta}:=\left(\begin{array}{cccr}
x_{n} & 0 & 0 & 0 \\
0 & x_{n} & 0 & 0 \\
0 & 0 & x_{n}^{\prime} & 0 \\
0 & 0 & 0 & x_{n}^{\prime}
\end{array}\right) x_{n}, x_{n}^{\prime} \in \mathbb{R}
$$

Then from the formula (2.2), it follows that $\left[P\left(\left\{\hat{x}_{n, \alpha}\right\}\right)\right]^{1 / 2}$ is a polynomial where each $x_{n}, x_{n}^{\prime}$ occurs at most once in each monomial!

To see this one has to take $-\psi_{n, \alpha}^{(1)} \mathscr{C}_{\alpha \beta} \hat{x}_{n, \beta} \psi_{n, \beta}^{(1)}$ as the "interaction"-term: the statement then follows from an expansion of the integrand in (2.2) and term by term integration à la Berezin.

For $x_{n}, x_{n}^{\prime}>0$, Proposition 3.2 implies that $\left[P\left(\left\{\hat{x}_{n, \alpha}\right\}\right)\right]^{1 / 2}$ is strictly positive. Also

$$
\left[P\left(\left\{\hat{x}_{n, \alpha}\right\}\right)\right]^{1 / 2}=\sum_{\varrho=1}^{2|\mathscr{T}|} \sum_{\substack{\Delta_{1}, \Delta_{2} \mathcal{C} \mathcal{T} \\\left|\Delta_{1}\right|+\left|\Delta_{2}\right|=\varrho}}\left[d_{\left\{\Delta_{1}, \Delta_{2}\right\}} \sum_{n \in \Delta_{1}} x_{n} \prod_{m \in \Delta_{2}} x_{m}^{\prime}\right] .
$$

Proposition 3.4. For all $\Delta_{1}, \Delta_{2} \subseteq \mathscr{T}$

$$
d_{\left\{\Delta_{1}, \Delta_{2}\right\}} \geqq 0 \text {. }
$$

Proof. As in Proposition 3.3

A similar argument shows that (3.14) also holds in the pseudoscalar case. This concludes the proof of the main theorem.

Remark. It is tempting to try to obtain a more explicit proof of the above statements by explicit computation of the coefficients $c_{\left\{\Delta_{\alpha}\right\}}, d_{\left\{\Delta_{\alpha}\right\}}$ à la Berezin; in order to do so one has to develop a set of "Feynman-rules" for latticedeterminants. By performing such a calculation the author has checked positivity for the first few terms in a "Wilson-expansion" [8] (=expansion of the kinetic term), but in higher orders the combinatorics becomes very involved so a proof to all orders will be extremely tedious if feasible at all.

\section{Discussion}

The range of applicability of the inequalities (1.3) is not yet clear. In four dimensions they are possibly not of much use in their present form if the purely scalar $A_{4}^{4}$-theory does not exist which appears to be likely $([9,10]$; however, there is as yet no complete proof of non-existence). In this case the inequalities at least ensure positivity of (pseudo)scalar Schwinger-functions. Nonetheless, it will be interesting to see what generalizations are possible and, in particular, whether similar inequalities exist in presence of both scalar and pseudoscalar interactions. Such a result would be very desirable for a thorough investigation of the 
supersymmetric $\phi^{3}$-model in the framework of multiplicative renormalization along the lines proposed by Schrader ${ }^{9}[10,11]$. Also, the possible existence of inequalities for lattice-gauge theories in four dimensions is an attractive speculation.

In less than four dimensions where the corresponding scalar theories are known to exist [12], the inequalities might be useful for the treatment of the Yukawa-model plus scalar interactions in two and three dimensions, respectively -in particular, it should be possible to gain some more insight into the supersymmetric $\phi^{3}$-model in two dimensions (which differs from its fourdimensional counterpart in field-content). To sketch another application that I have in mind consider a model with spontaneously broken symmetry: adding a term $a^{4} h \sum_{n \in \mathscr{T}} A_{n}$ to the action $(1.1)(h>0)$, one should find that in the infinite volume limit $([2,13] ; V=$ volume $)$

$$
\lim _{h \rightarrow+0} \lim _{\substack{V \rightarrow \infty \\ a>0}}\langle A\rangle_{0, h} \geqq \text { const }>0 .
$$

On account of inequality (1.3) $\langle A\rangle_{1, h} \geqq\langle A\rangle_{0, h}$ for any $h>0$ and $V<\infty$. Consequently, if the limit exists

$$
\lim _{h \rightarrow+0} \lim _{\substack{V \rightarrow \infty \\ a>0}}\langle A\rangle_{1, h} \geqq \text { const }>0 \text {. }
$$

Thus, if the purely scalar theory exhibits spontaneous symmetry breaking this situation persists if the Yukawa-interaction is turned on which, of course, is in accord with physical intuition.

After I had submitted this paper for publication L. Rosen and the referee kindly brought the following points to my attention:

1) For $d=2$ and Dirac-spinors, essentially the same results were obtained by Alan McDermot in his PhD-Thesis; his work is definitely prior to mine $[14,15]$. In his thesis, A. McDermot also proves the validity of the lattice approximation in $Y_{2}$.

2) When the fermions are Wick-ordered, the positivity property proven in Section 3 may cease to hold. This is not a problem in supersymmetric theories where Wick-ordering is unnecessary.

Also, I should like to point out that the results of this paper are independent of what $\nabla_{k n}^{\mu}$ one chooses for the fermions since all that has to be required is reality and antisymmetry of the matrix $\nabla_{k n}^{\mu}$.

Acknowledgments. It is a pleasure to thank Professor Wess for a useful conversation and encouragement.

\section{References}

1. Glimm,J., Jaffe, A.: A tutorial course in constructive QFT. In : Cargèse Summer School Lectures 1976

2. Simon,B.: The $P(\phi)_{2}$-(Euclidean) QFT, Princeton series in physics. Princeton: Princeton University Press 1974

Ginibre,J.: Commun. math. Phys. 16, 310 (1970)

9 In this context, supersymmetric theories are of particular interest because they relate bosonic and fermionic expectation-values via Ward-identities 
3. Osterwalder, K., Schrader, R. : Helv. Phys. Acta 46, 277 (1973)

4. Fröhlich,J., Osterwalder, K.: Helv. Phys. Acta 47, 781 (1974) Nicolai,H.: Unpublished

5. Berezin, F. A. : The method of second quantization. London and New York: Academic Press 1966

6. Matthews,P.T., Salam,A.: Nuovo Cimento 12, 563 (1954); 2, 120 (1955)

7. Zinn-Justin, J.: In: Trends in elementary particle theory, Bonn Summer School. Berlin-HeidelbergNew York: Springer 1974

8. Wilson, K.G.: Phys. Rev. D10, 2445 (1974)

9. Kogut,J., Wilson,K.G. : Phys. Rev. 12, 75 (1975)

10. Schrader, R.: Commun. math. Phys. 49, 131 (1976); 50, 97 (1976)

11. Nicolai,H.: Contributed paper to the proceedings of the Tübingen International Colloquium on group theoretical methods in physics (1977) Wess, J., Zumino, B. : Nucl. Phys. B70, 39 (1974)

12. Feldman, J., Osterwalder, K.: Ann. Phys. 97, 80 (1976)

13. Nelson,E.: Probability theory and Euclidean field theory. In: Constructive QFT. BerlinHeidelberg-New York: Springer 1973

14. McDermot, A.: A lattice approximation to the Euclidean $Y_{2}$ quantum field theory and a correlation inequality. Ph. D. Thesis, Cornell University (1976)

15. McDermot, A. : Notices Am. Math. Soc. 23 A, 480 (1976); Announcement 76T-A190

Communicated by A. Jaffe

Received September 3, 1977 